\title{
Symmetry Classifications of Differential Equations with Arbitrary Parameters
}

\author{
Bai Yushan
}

\begin{abstract}
It is very important to specify the forms of arbitrary parameters in differential equations. The procedure for performing this task is known as Lie symmetry classification. The main technique used to analyze the symmetry classification problems is the traditional Lie's algorithm. In this paper, symmetry classifications of KP equation and Hopf equation are given.
\end{abstract}

Index Terms-Differential equations, arbitrary parameters, Lie symmetry, symmetry classification.

\section{INTRODUCTION}

Many physical phenomena in the fields such as physics, fluid dynamics can be described by nonlinear partial differential equations (PDEs) [1], [2]. The theory of Lie symmetry groups of differential equations was developed by $\mathrm{S}$. Lie, which was called classical Lie method. Nowadays, Lie symmetry method has been widely used in diverse fields of mathematics and many areas of physics [3]-[6]. Determining the group invariant solutions, construction of new solutions for the system from the known ones, group classification of PDEs, reduction of the order of ordinary differential equations, and mapping solutions to other solutions are the important applications of classical Lie method in the theory of differential equations[6]-[12]. Symmetry classification of PDEs with arbitrary parameters (or functions) is one of the main applications of symmetry method to differential equations. For a family of PDEs with arbitrary parameter $\rho$, finding both the parameters $\rho$ and corresponding maximal set of symmetries $\Omega_{\rho}$ is called the symmetry classification problem of the family of PDEs [14]-[18]. The main technique used to analysis the symmetry classification of PDEs with arbitrary parameters is the Lie's algorithm.

Consider a general form kth-order PDEs with parameter $\rho$ :

$$
F^{\delta}(E)=0, \delta=1, \cdots, S
$$

where $x=\left(x^{1}, \cdots, x^{n}\right)$ denotes $n$ independent variables, $u=\left(u^{1}, \cdots, u^{m}\right)$ denotes the dependent variables, and $u_{(i)}$ denotes the set of $u$ with respect to $x$ and

Manuscript received October 29, 2013; revised January 13, 2014. The work was financially supported by the Natural Science Foundation of Inner Mongolia (No.2011BS0106)

Bai Yushan is with the College of Sciences of Inner Mongolian University of Technology Hohhot 010051, P.R.C. (e-mail: mbaiyushan@imut.edu.cn).

$$
E=\left(x, u, u_{(1)}, \cdots, u_{(k)}, \rho\right)
$$

The generator of the symmetry of PDEs (1) is decomposed to [2]

$$
X=\xi^{i}(x, u) \frac{\partial}{\partial x_{i}}+\eta^{\alpha}(x, u) \frac{\partial}{\partial u^{\alpha}} .
$$

We will write the $k$-th prolongation of $X$ to the derivatives involved in the system (1) in the following form

$$
\tilde{X}=X+\eta_{\alpha}^{J}\left(x, u_{(k)}\right) \frac{\partial}{\partial u_{J}^{\alpha}}
$$

where $J=\left(j_{1}, \cdots, j_{n}\right)$, with $|J|=\sum_{i=1}^{n} j_{i} \leq k$, $u_{J}^{\alpha}=u_{j_{1} j_{2} \cdots j_{n}}^{\alpha}=\frac{\partial^{|J|} u^{\alpha}}{\partial x_{1}^{j_{1}} \partial x_{2}^{j_{2}} \cdots \partial x_{n}^{j_{n}}}$. The coefficient $\eta_{\alpha}^{J}(l=0,1)$ are given by

$\eta_{\alpha}^{J}\left(x, u^{(n)}\right)=D_{J}\left(\eta^{\alpha}-\xi^{q} u_{q}^{\alpha}\right)+\xi^{q} u_{J, q}^{\alpha}, \alpha=1, \cdots, m$,

where $u_{i}^{\alpha}=\frac{\partial u^{\alpha}}{\partial x_{i}}$, and $u_{J, i}^{\alpha}=\frac{\partial u_{J}^{\alpha}}{\partial x_{i}}$.

The total derivative

$$
D_{i}=\frac{\partial}{\partial x_{i}}+u_{J, i}^{\alpha} \frac{\partial}{\partial u_{J}^{\alpha}}, D_{i}^{j_{i}}=\underbrace{D_{i} D_{i} \cdots D_{i}}_{j_{i} \text { times }}, D_{J}=D_{1}^{j_{1}} D_{2}^{j_{2}} \cdots D_{n}^{j_{n}} .
$$

The system (1) is invariant under the symmetry group with the generator (2) if and only if

$$
\tilde{X} F^{\delta}(E)=0,
$$

when (1) is held.

One then reads off the coefficients of the different monomials in the derivatives of $u$ in (4) and setting those to zero yields a linear over-determined PDEs, called the determining equations (DTEs) denoted as $D(\rho)=0$, satisfied by $\mathrm{X}$ and which determine operator $\mathrm{X}$.

In the above algorithm, admitting symmetry of PDEs(1) means that the determining equation $D(\rho)=0$ is solvable.

Thus, the question of the group classification is completely transformed into solving the parameterized determining equations. It is known that the over-determined PDEs $D(\rho)=0$ is not always solvable for every $\rho$. Thus, we have to find the proper conditions for $\rho$ so that the equation 
$D(\rho)=0$ is solvable. The conditions are usually given by the so called classifying equations, which are satisfied only by $\rho$. The key point to determine the symmetry classification is to find out classification equations and solve determining equations.

\section{SYMmetry CllassifiCATION OF PDES}

\section{A. The Perturbed KP Equation}

The KP equation is given by

$$
\left(u_{t}+f(u)^{\sigma} u_{x}+u_{x x x}\right)_{x}+g(x) u_{y y}=0,
$$

where $f(u), g(x)$ are arbitrary functions.

Firstly, we seek a symmetry generator

$$
\mathrm{X}=\xi \frac{\partial}{\partial x}+\mu \frac{\partial}{\partial t}+\tau \frac{\partial}{\partial y}+\eta \frac{\partial}{\partial u}
$$

of equation (7), where $\xi, \tau, \mu$ and $\eta$ depend on variables $x, t, y$ and $u$.

Form (4), we obtain following determining equations

$$
\begin{gathered}
\xi_{u}=\mu_{u}=\tau_{u}=\eta_{u u}=\mu_{x}=\mu_{y}=\tau_{x}=\eta_{x u}=0, \\
2 f(u)^{2} g(x) \eta_{y u}-f(u)^{2} g(x) \tau_{y y}=0, \\
f(u)^{2} g(x) \eta_{y y}+f(u)^{2} \eta_{x t}+f(u)^{2+\sigma} \eta_{x x} \\
+f(u)^{2} \eta_{x x x x}, 4 f(u)^{2} \eta_{x u}-6 f(u)^{2} \xi_{x x}=0 \\
-2 f(u)^{2} g(x) \xi_{y}-f(u)^{2} \tau_{t}=0, \\
-f(u)^{2} \mu_{t}+3 f(u)^{2} \xi_{x}, 4 f(u)^{2} g(x) \xi_{x} \\
-2 f(u)^{2} g(x) \tau_{y}+f(u)^{2} \xi g^{\prime}(x)=0, \\
6 f(u)^{2} \eta_{x x u}-f(u)^{2} \xi_{t}+2 f(u)^{2+\sigma} \xi_{x} \\
-4 f(u)^{2} \xi_{x x x}+\sigma f(u)^{1+\sigma} \eta_{x x x x} f(u)=0, \\
f(u)^{2} \eta_{t u}+2 f(u)^{2+\sigma} \eta_{x u}+4 f(u)^{2} \eta_{x x x u} \\
-f(u)^{2} g(x) \xi_{y y}-f(u)^{2} \xi_{x t}-f(u)^{2+\sigma} \xi_{x x} \\
-f(u)^{2} \xi_{x x x x}+2 \sigma f(u)^{1+\sigma} \eta_{x} f^{\prime}(u)=0, \\
\sigma f(u)^{1+\sigma} \eta_{u} f^{\prime}(u)+2 \sigma f(u)^{1+\sigma} \xi_{x} f^{\prime}(u) \\
-\sigma f(u)^{\sigma} \eta f^{\prime}(u)^{2}+\sigma^{2} f(u)^{\sigma} \eta f^{\prime}(u)^{2} \\
+\sigma f(u)^{1+\sigma} \eta f^{\prime \prime}(u)=0 .
\end{gathered}
$$

Solving this system we obtain

$$
\mathrm{X}=c_{1} \frac{\partial}{\partial t}+c_{2} \frac{\partial}{\partial y}
$$

and classifying equations

$$
\begin{aligned}
& f^{\prime}(u)=0, g^{\prime}(x)=0, \\
& -f^{\prime}(u)^{2}+\sigma f^{\prime}(u)^{2}+f(u) f^{\prime \prime}(u)=0, \\
& g^{\prime}(x)^{2} g^{\prime \prime}(x)-2 g(x) g^{\prime \prime}(x)^{2}+g(x) g^{\prime}(x) g^{\prime \prime \prime}(x)=0
\end{aligned}
$$

for arbitrary functions $f(u), g(x)$.

Then the solutions of the classifying equations lead to the following cases.

1) $f(u)=F, g(x)=G$, where $F, G$ are constants.

$$
\begin{aligned}
& \mathrm{X}=\left(A_{2} x+A_{3} y+2 F A_{2} t+A_{4}\right) \frac{\partial}{\partial x} \\
& +\left(3 A_{2} t+A_{5}\right) \frac{\partial}{\partial t}+\left(2 A_{2} y-2 G A_{3} t+A_{5}\right) \frac{\partial}{\partial y} \\
& +\left(A_{1} u+m(x, t, y)\right) \frac{\partial}{\partial u},
\end{aligned}
$$

where function $m(x, t, y)$ satisfies equation

$$
\left(m_{t}+F m_{x}+m_{x x x}\right)_{x}+G m_{y y}=0 .
$$

2) $f(u)=F, g(x)=x^{\theta}, \theta \neq-1$.

$$
\mathrm{X}=A_{2} \frac{\partial}{\partial t}+A_{3} \frac{\partial}{\partial y}+\left(A_{1} u+m(x, t, y)\right) \frac{\partial}{\partial u},
$$

where function $m(x, t, y)$ satisfies equation

$$
\left(m_{t}+F m_{x}+m_{x x x}\right)_{x}+x^{\theta} m_{y y}=0 .
$$

- $g(x)=\frac{1}{x}$.

$$
X=A_{2} \frac{\partial}{\partial t}+A_{3} \frac{\partial}{\partial y}+\left(A_{1} u+m(x, t, y)\right) \frac{\partial}{\partial u},
$$

where function $m(x, t, y)$ satisfies equation $\left(m_{x t}+F m_{x x}+m_{x x x x}\right)_{x}+\frac{1}{x} m_{y y}=0$.

3) $f(u)=F, g(x)=e^{x}$.

$$
\mathrm{X}=A_{3} \frac{\partial}{\partial x}+A_{2} \frac{\partial}{\partial t}+\left(\frac{1}{2} A_{3} y+A_{4}\right) \frac{\partial}{\partial y}+\left(A_{1} u+m(x, t, y)\right) \frac{\partial}{\partial u},
$$

where function $m(x, t, y)$ satisfies equation

$\left(m_{t}+F m_{x}+m_{x x x}\right)_{x}+e^{x} m_{y y}=0$.

4) $f(u)=u^{\frac{1}{\sigma}}, g(x)=G$, here $G$ is constant.

$$
\begin{aligned}
& \mathrm{X}=\left(\frac{1}{3} A_{1} x-\frac{\tilde{k}^{\prime}(t)}{2 G}+\tilde{m}(t)\right) \frac{\partial}{\partial x}+ \\
& \left(A_{1} t+A_{2}\right) \frac{\partial}{\partial t}+\left(\frac{2}{3} A_{1} y+\tilde{k}(t)\right) \frac{\partial}{\partial y} \\
& +\left(-\frac{2}{3} A_{1} u-\frac{\tilde{k}^{\prime \prime}(t)}{2 G} y+\tilde{m}^{\prime}(t)\right) \frac{\partial}{\partial u},
\end{aligned}
$$


where $\tilde{k}(t), \tilde{m}(t)$ are arbitrary functions.

5) $f(u)=u^{\frac{1}{\sigma}}, g(x)=x^{\theta}, \theta \neq-1,-4$.

$$
\begin{aligned}
& X=A_{1} x \frac{\partial}{\partial x}+\left(3 A_{1} t+A_{2}\right) \frac{\partial}{\partial t} \\
& +\left(\left(2 A_{1}+\frac{\theta A_{1}}{2}\right) y+A_{3}\right) \frac{\partial}{\partial y} \\
& -2 A_{1} u \frac{\partial}{\partial u} .
\end{aligned}
$$

- $g(x)=\frac{1}{x}$.

$$
\begin{aligned}
& X=A_{1} \frac{\partial}{\partial x}+\left(3 A_{1} t+A_{2}\right) \frac{\partial}{\partial t} \\
& +\left(\frac{3}{2} A_{1} y+A_{3}\right) \frac{\partial}{\partial y}-2 A_{1} u \frac{\partial}{\partial u} .
\end{aligned}
$$

- $g(x)=\frac{1}{x^{4}}$.

$$
\begin{aligned}
& X=A_{1} \frac{\partial}{\partial x}+\left(3 A_{1} t+A_{3}\right) \frac{\partial}{\partial t} \\
& +A_{2} \frac{\partial}{\partial y}-2 A_{1} u \frac{\partial}{\partial u} .
\end{aligned}
$$

6) $f(u)=u^{\frac{1}{\sigma}}, g(x)=e^{x}$.

$$
X=A_{1} \frac{\partial}{\partial x}+A_{2} \frac{\partial}{\partial t}+\left(\frac{A_{1}}{2} y+A_{3}\right) \frac{\partial}{\partial y} .
$$

7) $f(u)=F, g(x)$ is an arbitrary function, $F$ is constant.

$$
X=A_{1} \frac{\partial}{\partial t}+A_{2} \frac{\partial}{\partial y}+\left(A_{3} u+m(x, t, y)\right) \frac{\partial}{\partial u},
$$

where function $m(x, t, y)$ satisfies equation

$$
\left(m_{t}+F m_{x}+m_{x x x}\right)_{x}+g(x) m_{y y}=0 .
$$

8) $f(u)=u^{\frac{1}{\sigma}}, g(x)$ is an arbitrary function.

$$
X=A_{1} \frac{\partial}{\partial t}+A_{2} \frac{\partial}{\partial y} .
$$

Here $A_{j}, j=1, \cdots, 3$ are arbitrary constants.

\section{B. Hopf Equation}

Consider Hopf equation

$$
u_{t}+u u_{x}=\left(K(u) u_{x}\right)_{x}
$$

where $K(u)$ is an arbitrary function.

Introducing potential variable $v$, we get following potential system [12]

$$
\begin{aligned}
& v_{x}=u \\
& v_{t}=-\frac{1}{2} u^{2}+K(u) u_{x}
\end{aligned}
$$

which is equivalent to original equation (9).

Let

$$
X=\xi \frac{\partial}{\partial x}+\mu \frac{\partial}{\partial t}+\varphi \frac{\partial}{\partial u}+\eta \frac{\partial}{\partial v}
$$

be the symmetry generator of system (6), where $\xi, \mu, \varphi$ and $\eta$ are unknown functions of four variables $x, t, y$ and $u$.

The following is to determine functions $\xi, \mu, \varphi$ and $\eta$ and $K(u)$ and show whether or not the original equation (5) admits potential symmetries. i.e., at least one of the functions $\xi, \mu, \varphi$ depends on potential variable $v$.

1) $K(u)$ is an arbitrary function.

$$
X=A_{1} \frac{\partial}{\partial x}+A_{2} \frac{\partial}{\partial t}+A_{3} \frac{\partial}{\partial v}
$$

2) $K(u)=K$.

$$
\begin{aligned}
& X=\left(c_{1} x t+c_{2} x+c_{3} t+c_{4}\right) \frac{\partial}{\partial x} \\
& +\left(c_{1} t^{2}+2 c_{2} t+c_{5}\right) \frac{\partial}{\partial t} \\
& +\left(\left(-c_{1} t-c_{2}\right) u+c_{1} x+c_{3}\right) \frac{\partial}{\partial u} \\
& +\left(\frac{1}{2} c_{1} x^{2}+c_{3} x+K c_{1} t+c_{6}\right) \frac{\partial}{\partial v} .
\end{aligned}
$$

3) $K(u)=\frac{K}{u^{2}}, K=$ costant.

$$
\begin{aligned}
& \mathrm{X}=\left(-3 A_{1}+A_{2}\right) \frac{\partial}{\partial x}+\left(-4 A_{1}+A_{3}\right) \frac{\partial}{\partial t} \\
& +A_{1} u \frac{\partial}{\partial u}+\left(-2 A_{1}+A_{4}\right) \frac{\partial}{\partial v}
\end{aligned}
$$

4) $K(u)=K_{1} u+K_{2}$.

$$
\begin{aligned}
& X=\left(A_{1} K_{2} t+A_{2}\right) \frac{\partial}{\partial x}+\left(-A_{1} K_{1} t+A_{3}\right) \frac{\partial}{\partial t} \\
& +\left(A_{1} K_{1} u+A_{1} K_{2}\right) \frac{\partial}{\partial u}+\left(A_{1} K_{1} v+A_{1} K_{2} x+A_{4}\right) \frac{\partial}{\partial v}
\end{aligned}
$$

5) $K(u)=K_{3} e^{K_{4} u+K_{5}}$

$$
\begin{aligned}
& X=\left(A_{1} K_{4} x+A_{1} t+A_{2}\right) \frac{\partial}{\partial x}+\left(A_{1} K_{4} t+A_{3}\right) \frac{\partial}{\partial t} \\
& +A_{1} \frac{\partial}{\partial u}+\left(A_{1} x+A_{1} K_{4} v+A_{4}\right) \frac{\partial}{\partial v}
\end{aligned}
$$


Here $K_{i}, i=1, \cdots, 5, \quad$ and $\quad A_{r}, r=1, \cdots, 4 \quad$ are arbitrary constants.

\section{CONCLUSION}

Lie's algorithm is the main technique used to analyze the symmetry classification of differential equations with arbitrary parameters. The key point to determine the symmetry classification is to find out classification equations and solve determining equations. Symmetry method has many applications, including the construction of analytic solutions of nonlinear differential equations, the classification of such equations, the construction of conservation laws, testing of numerical computations etc [19]-[26].

\section{REFERENCES}

[1] P. J. Olver, Application of Lie groups to differential equations, New York: Springer-Verlag, ch. 1, 1993.

[2] N. H. Ibragimov, Elementary Lie Group Analysis and Ordinary Differential Equations, Chichester: Wiley, ch. 1, pp. 3-23, 1999.

[3] L. V. Ovsiannikov, "Groups and group-invariant solutions of partial differential equations," Dokl. RAS, vol. 3, pp. 439-442, 1958.

[4] L. V. Ovsiannikov, Group properties of differential equations, Moscow: Novosibirsk, 1962, ch. 3, pp. 92-137.

[5] W. F. Ames, "Some exact solutions for wave propagation in viscoelastic and electrical transmission," Int. J. Nonlinear Mech., vol. 17, pp. 223-230, 1982.

[6] L. V. Ovsiannikov, Group analysis of differential equations, New York: Academic Press, 1982, ch. 3, pp. 92-137.

[7] N. H. Ibragimov, CRC Handbook of Lie Group Analysis of Differential Equations, Boca Raton FL: CRC Press, vo1. 1, 1994, ch.1, pp. 36-38.

[8] N. H. Ibragimov, CRC Handbook of Lie Group Analysis of Differential Equations, Boca Raton FL: CRC Press, vo1. 2, 1995, ch.2, pp. 30-35.

[9] G. W. Bluman and S. Kumei, Symmetries and differential Equations, New York: Springer, 1989, ch. 4.

[10] N. H. Ibragimov, CRC Handbook of Lie Group Analysis of Differential Equations, Boca Raton FL: CRC Press, vo1.3, 1996, ch. 8.

[11] T. Chaolu and Y.-S. Bai, "Differential Characteritic Set Alogorithm for the Complete Symmetry Classification of (partial) Differential Equations," Applied Mathematics And Mechanics, vol. 32, pp. 556-566, 2003.

[12] G. I. Burde, "Potential symmetries of the wave equation $u_{t t}=u u_{x(x)}$ and relared exact and approximate solutions," J.Phys.A:Math.Gen., vol. 34, pp. 5355-5371, July 2001.
[13] S. Kumi and G. Bluman, "When nonlinear differential equations are equivalent to linear differential equations," SIAM J. Appl. Math, vol. 42, pp. 1157-1173, 1982.

[14] Q. U. Changzhneng, "Allowed transformations and symmetry classes of variable coefficient Burgers equations," IMA. J. Appl. Math, vol. 54, pp. 203-225, 1995.

[15] M. Yürüsoy, "Group classification of unsteady boundary layer equations of a class of non-newtonian fluids," J. Appl. Math.Com., vol. 150, pp. 775-783, 2004.

[16] M. Pakdemirli, M. Yurusoy, and A. Kuukbursa, "Symmetry groups of boundary layer equations of a class of non-Newtonian fluids," Int. J. Non-Linear Mech, vol. 31, pp. 267-276, 1996.

[17] P. J. Olver and P. Rosenau, "The construction of special solutions to partial differential equations," Phys. Lett. A., vol. 114, pp. 107-112, Feb. 1986.

[18] E. Pucci and G. Saccomandi, "On the weak symmetry groups of partial differential equations,” J. Math. Anal. Appl., vol. 163, pp. 588-598, Jan. 1992.

[19] W. I. Fushchlicch, "Conditional symmetries of the equations of mathematical physics," J. Ukrain Math, vol. 43, pp. 1456-1470, 1991.

[20] S. Y. Lou, "Symmetries of the KdV equation and four hierarchies of the integrodifferential KdV equations," J. Math. Phys, vol. 35, pp. 2390-2396, 1994.

[21] P. A. Clarkson and M. D. Kruskal, "New similarity reductions of the Boussinesq equation,” J. Math. Phys., vol. 30, pp. 2201-2207, 1989.

[22] S. Y. Lou, "A note on the new similarity reductions of the Boussinesq equation,” Phys. lett. A., vol. 151, pp. 133-135, 1990.

[23] S.Y. Lou, H.Y. Ruan, and D. F. Chen, "Similarity reductions of the KP equation by a direct method," J. Phys. A,Math. Gen., vol. 24, pp. 1455-1467, 1991.

[24] S. Y. Lou and H. Y. Ruan, "Nonclassical analysis and Painleve property for the Kupershmidt equations," J. Phys. A, vol. 26, pp. 4679-4688, 1993.

[25] J. Pucci, "Similarity reductions of partial differential equations," J.Phys. A: Math. Gen, vol. 25, pp. 2631-2640, 1992.

[26] G. W. Bluman et al., "Local and nonlocal symmetries for nonlinear telegraph equations," J Math Phys., vol. 46, pp. 1-12, 2005.

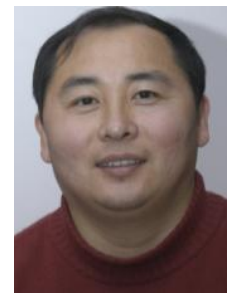

Bai Yushan was born in April 1974, in Tongliao, Inner Mongolia Autonomous Region of China. He received the bachelor degree in mathematics education from Inner Mongolian Normal University in 1998. He received the master degree and doctor degree in solid mechanics from Inner Mongolian University of Technology in 2004, 2010, respectively. Now he is an associate professor of mathematics in College of Sciences of Inner Mongolian University of Technology. His research interests are in symmetry analysis of differential equations, Mathematics Mechanization and integrable systems and published more than 10 articles in recent years. 